\title{
Affine and toric arrangements
}

\author{
Richard Ehrenborg, Margaret Readdy, and Michael Slone
}

Department of Mathematics, University of Kentucky, Lexington, KY 40506-0027

\begin{abstract}
We extend the Billera-Ehrenborg-Readdy map between the intersection lattice and face lattice of a central hyperplane arrangement to affine and toric hyperplane arrangements. For toric arrangements, we also generalize Zaslavsky's fundamental results on the number of regions.
\end{abstract}

Résumé. Nous étendons l'opérateur de Billera-Ehrenborg-Readdy entre la trellis d'intersection et la trellis de faces d'un arrangement hyperplans centrals aux arrangements affines et toriques. Pour les arrangements toriques, nous généralisons aussi les résultats fondamentaux de Zaslavsky sur le nombre de régions.

Keywords: toric arrangement, hyperplane arrangement, characteristic polynomial, coalgebra

\section{Introduction}

Traditionally combinatorialists have studied topological objects that are spherical, such as polytopes, or which are homeomorphic to a wedge of spheres, such as those obtained from shellable complexes. In this paper we break from this practice and study hyperplane arrangements on the $n$-dimensional torus.

It is classical that the convex hull of a finite collection of points in Euclidean space is a polytope and its boundary is a sphere. The key ingredient in this construction is convexity. At the moment there is no natural analogue of this process to obtain a complex whose geometric realization is a torus.

In this paper we are taking a zonotopal approach to working with arrangements on the torus. Recall that every central hyperplane arrangement gives rise to a zonotope, that is, a spherical object. By considering an arrangement on the torus, we are able to obtain a subdivision whose geometric realization is indeed the torus. This amounts to restricting ourselves to arrangements whose subspaces in the Euclidean space $\mathbb{R}^{n}$ have coefficient matrices with rational entries. Under the quotient map $\mathbb{R}^{n} \longrightarrow \mathbb{R}^{n} / \mathbb{Z}^{n}=T^{n}$ these subspaces are sent to subtori of the $n$-dimensional torus $T^{n}$.

Zaslavsky initiated the modern study of hyperplane arrangements in his fundamental treatise [43]. For early work in the field, see the references given in Grünbaum's text [26, Chapter 18]. Zaslavsky showed that evaluating the characteristic polynomial of a central hyperplane arrangement at -1 gives the number of regions in the complement of the arrangement. For central hyperplane arrangements, Bayer and Sturmfels proved the flag $f$-vector of the arrangement can be determined from the intersection lattice [6]; see Theorem 2.3. Billera, Ehrenborg and Readdy proved that the flag $f$-vector of the arrangement can be determined from the flag $f$-vector of the intersection lattice. Recall that the cd-index of a regular cell complex is an efficient tool to encode its flag $f$-vector without linear redundancies [5]. The Billera-Ehrenborg-Readdy theorem gives an explicit way to compute the cd-index of the arrangement [8].

The first step is to generalize Zaslavsky's theorem on the number of regions of a hyperplane arrangement to the toric case. Although there is no intersection lattice, one works with the intersection poset. From the Zaslavsky result we obtain a toric version of the Bayer-Sturmfels result for hyperplane arrangements, that is, there is a natural poset map from the face poset to the intersection poset and the cardinality of the inverse image of a chain under this map is described.

As in the case of a central hyperplane arrangement, our toric version of the Bayer-Sturmfels result determines the flag $f$-vector of the face poset of a toric arrangement in terms of its intersection poset. However, this is far from being explicit. Using the coalgebraic techniques from [18], we are able to determine the flag $f$-vector explicitly in terms of the 
flag $f$-vector of the intersection poset. Moreover, the answer is given by a cd type of polynomial. The flag $f$-vector of a regular spherical complex is encoded by the cd-index, a non-commutative polynomial in the variables $\mathbf{c}$ and $\mathbf{d}$, whereas the $n$-dimensional toric analogue is a cd-polynomial plus the $\mathbf{a b}$-polynomial $(\mathbf{a}-\mathbf{b})^{n+1}$.

Zaslavsky also showed that evaluating the characteristic polynomial of an affine arrangement at 1 gives the number of bounded regions in the complement of the arrangement. Thus we return to affine arrangements in Euclidean space with the twist that we study the unbounded regions. The unbounded regions form a spherical complex. In the case of central arrangements, this complex is exactly what was studied previously by Billera, Ehrenborg and Readdy [8]. For non-central arrangements, we determine the cd-index of this complex in terms of the lattice of unbounded intersections of the arrangement.

Interestingly, the techniques for studying toric arrangements and the unbounded complex of non-central arrangements are similar. Hence, we present these results in the same paper. For example, the toric and non-central analogues of the Bayer-Sturmfels theorem only differ in which Zaslavsky invariant is used. The coalgebraic translations of the two analogues involve exactly the same argument, and the resulting underlying maps $\varphi_{t}$ (in the toric case) and $\varphi_{u b}$ (in the non-central case) only differ slightly in their definitions.

We end with many open questions about subdivisions of manifolds.

\section{Preliminaries}

All the posets we will work with are graded, that is, posets having a unique minimal element $\hat{0}$, a unique maximal element $\hat{1}$, and rank function $\rho$. For two elements $x$ and $z$ in a graded poset $P$ such that $x \leq z$, let $[x, z]$ denote the interval $\{y \in P: x \leq y \leq z\}$. Observe that the interval $[x, z]$ is itself a graded poset. For standard poset terminology, we refer the reader to Stanley's work [37].

We now review important results about hyperplane arrangements, the cd-index and coalgebraic techniques that are essential for proving the main results of this paper.

\subsection{Hyperplane arrangements}

Let $\mathcal{H}=\left\{H_{1}, \ldots, H_{m}\right\}$ be a hyperplane arrangement in $\mathbb{R}^{n}$, that is, a finite collection of affine hyperplanes in $n$ dimensional Euclidean space $\mathbb{R}^{n}$. For brevity, throughout this paper we will often refer to a hyperplane arrangement as an arrangement. We call the arrangement essential if the normal vectors to the hyperplanes in $\mathcal{H}$ span $\mathbb{R}^{n}$. In this paper we are only interested in essential arrangements.

Observe that the intersection $\bigcap_{i=1}^{m} H_{i}$ of all of the hyperplanes in an essential arrangement is either the empty set $\emptyset$ or a singleton point. We call an arrangement central if the intersection of all the hyperplanes is one point. We may assume that this point is the origin $\mathbf{0}$ and hence all of the hyperplanes are codimension 1 subspaces. If the intersection is the empty set, we call the arrangement non-central.

The intersection lattice $\mathcal{L}$ is the lattice formed by ordering all the intersections of hyperplanes in $\mathcal{H}$ by reverse inclusion. If the intersection of all the hyperplanes in a given arrangement is empty, then we include the empty set $\emptyset$ as the the maximal element in the intersection lattice. If the arrangement is central the maximal element is $\{\mathbf{0}\}$. In all cases, the minimal element of $\mathcal{L}$ will be all of $\mathbb{R}^{n}$.

For a hyperplane arrangement $\mathcal{H}$ with intersection lattice $\mathcal{L}$ its characteristic polynomial is defined by

$$
\chi(\mathcal{H} ; t)=\sum_{\substack{x \in \mathcal{C} \\ x \neq \emptyset}} \mu(\hat{0}, x) \cdot t^{\operatorname{dim}(x)},
$$

where $\mu$ denotes the Möbius function. The characteristic polynomial is a combinatorial invariant of the arrangement. The fundamental result of Zaslavsky [43] is that this invariant determines the number and type of regions.

Theorem 2.1 (Zaslavsky) For a hyperplane arrangement $\mathcal{H}$ in $\mathbb{R}^{n}$ the number of regions is $(-1)^{n} \cdot \chi(\mathcal{H} ;-1)$. Furthermore, the number of bounded regions is given by $(-1)^{n} \cdot \chi(\mathcal{H} ; 1)$. 
For a graded poset $P$, define the two Zaslavsky invariants $Z$ and $Z_{b}$ by

$$
\begin{aligned}
& Z(P)=\sum_{\hat{0} \leq x \leq \hat{1}}(-1)^{\rho(x)} \cdot \mu(\hat{0}, x), \\
& Z_{b}(P)=(-1)^{\rho(P)} \cdot \mu(P) .
\end{aligned}
$$

In order to work with Zaslavsky's result, we need the following reformulation of Theorem 2.1.

Theorem 2.2 (i) For a central hyperplane arrangement $\mathcal{H}$ the number of regions is given by $Z(\mathcal{L})$, where $\mathcal{L}$ is the intersection lattice of the arrangement $\mathcal{H}$.

(ii) For a non-central hyperplane arrangement $\mathcal{H}$ the number of regions is given by $Z(\mathcal{L})-Z_{b}(\mathcal{L})$, where $\mathcal{L}$ is the intersection lattice of the arrangement $\mathcal{H}$. The number of bounded regions is given by $Z_{b}(\mathcal{L})$.

Given a central hyperplane arrangement $\mathcal{H}$ there are two associated lattices, namely the intersection lattice $\mathcal{L}$ and the lattice $T$ of faces of the arrangement. The lattice of faces can be seen as the face poset of the $C W$-complex obtained by intersecting the arrangement $\mathcal{H}$ with a sphere of radius $R$ centered at the origin. Each hyperplane corresponds to a great circle on the sphere. An alternative way to view the lattice of faces $T$ is that the dual lattice $T^{*}$ is the face lattice of the associated zonotope.

Let $\mathcal{L} \cup\{\hat{0}\}$ denote the intersection lattice with a new minimal element $\hat{0}$ adjoined. Define an order- and rank-preserving map $z$ from the dual lattice $T^{*}$ to the augmented lattice $\mathcal{L} \cup\{\hat{0}\}$ by sending a face of the arrangement, that is, a cone in $\mathbb{R}^{n}$, to its affine hull. Note that under the map $z$ the minimal element of $T^{*}$ is mapped to the minimal element of $\mathcal{L} \cup\{\hat{0}\}$. We view $z$ as a map from the set of chains of $T^{*}$ to the set of chains of $\mathcal{L} \cup\{\hat{0}\}$. Bayer and Sturmfels [6] proved the following result about the inverse image of a chain under the map $z$.

Theorem 2.3 (Bayer-Sturmfels) Let $\mathcal{H}$ be a central hyperplane arrangement with intersection lattice $\mathcal{L}$. Let $c$ be the chain $\left\{\hat{0}=x_{0}<x_{1}<\cdots<x_{k}=\hat{1}\right\}$ in $\mathcal{L} \cup\{\hat{0}\}$. Then the cardinality of the inverse image of the chain $c$ under the map $z: T^{*} \longrightarrow \mathcal{L} \cup\{\hat{0}\}$ is given by the product

$$
\left|z^{-1}(c)\right|=\prod_{i=2}^{k} Z\left(\left[x_{i-1}, x_{i}\right]\right) .
$$

\subsection{The cd-index and coalgebraic techniques}

Let $P$ be a graded poset of rank $n+1$ with rank function $\rho$ and let $\mathbf{a}$ and $\mathbf{b}$ be two non-commutative variables. The flag $f$-vector is defined as follows. For $S=\left\{s_{1}<\cdots<s_{k-1}\right\}$ a subset of $\{1, \ldots, n\}$ define $f_{S}$ to be the number of chains $c$ that have elements with ranks in the set $S$, that is, $f_{S}=\left|\left\{c: \rho\left(x_{1}\right)=s_{1}, \ldots, \rho\left(x_{k-1}\right)=s_{k-1}\right\}\right|$. The flag $h$-vector is obtained by the relation (here we also present its inverse)

$$
h_{S}=\sum_{T \subseteq S}(-1)^{|S-T|} \cdot f_{T} \quad \text { and } \quad f_{S}=\sum_{T \subseteq S} h_{T} .
$$

For $S$ a subset of $\{1, \ldots, n\}$ let $u_{S}$ be the monomial $u_{S}=u_{1} \cdots u_{n}$ where $u_{i}=\mathbf{b}$ if $i \in S$ and $u_{i}=\mathbf{a}$ if $i \notin S$. Then the $\mathbf{a b}$-index is given by the sum

$$
\Psi(P)=\sum_{S \subseteq\{1, \ldots, n\}} h_{S} \cdot u_{S}
$$

A poset $P$ is Eulerian if every interval $[x, y]$, where $x<y$, satisfies the Euler-Poincaré relation, that is, there are the same number of elements of odd as even rank. Equivalently, the Möbius function of $P$ is given by $\mu(x, y)=(-1)^{\rho(x, y)}$ for all $x \leq y$ in $P$. The quintessential result is that the ab-index of Eulerian posets has the following form.

Theorem 2.4 The $\mathbf{a b}$-index of an Eulerian poset $P$ can be expressed in terms of the noncommutative variables $\mathbf{c}=\mathbf{a}+\mathbf{b}$ and $\mathbf{d}=\mathbf{a b}+\mathbf{b a}$. 
This theorem was originally proved for face lattices of convex polytopes by Bayer and Klapper [5]. Stanley provided a proof for all Eulerian posets [39]. There are proofs which have both used and revealed the underlying algebraic structure. See for instance [14, 21]. When the $\mathbf{a b}$-index $\Psi(P)$ is written in terms of $\mathbf{c}$ and $\mathbf{d}$, the resulting polynomial is called the cd-index. There are linear relations holding among the entries of the flag $f$-vector of an Eulerian poset, known as the generalized Dehn-Sommerville relations; see [3]. The importance of the cd-index is that it removes all of these linear redundancies among the flag $f$-vector entries.

For a graded poset $P$ define $P^{*}$ to be the dual poset, that is, the poset having the same underlying set as $P$ but with the order relation reversed: $x<_{P^{*}} y$ if and only if $y<_{P} x$. Define the reverse of an ab-monomial $u=u_{1} u_{2} \cdots u_{n}$ to be $u^{*}=u_{n} \cdots u_{2} u_{1}$ and extend by linearity to an involution on $\mathbb{Z}\langle\mathbf{a}, \mathbf{b}\rangle$. Since $\mathbf{c}^{*}=\mathbf{c}$ and $\mathbf{d}^{*}=\mathbf{d}$, this involution applied to a cd-monomial reverses the cd-monomial. Finally, for a graded poset $P$ we have $\Psi(P)^{*}=\Psi\left(P^{*}\right)$.

A coproduct $\Delta$ on a module $C$ is a linear map $\Delta: C \longrightarrow C \otimes C$. We say that the coproduct is coassociative if $(\Delta \otimes$ id $) \circ \Delta=(\operatorname{id} \otimes \Delta) \circ \Delta$. We use the Sweedler notation [42] for the coproduct, writing

$$
\Delta(w)=\sum_{w} w_{(1)} \otimes w_{(2)} .
$$

The Sweedler notation for the $k$-ary coproduct is

$$
\Delta^{k-1}(w)=\sum_{w} w_{(1)} \otimes w_{(2)} \otimes \cdots \otimes w_{(k)} .
$$

Define a coproduct $\Delta$ on the algebra $\mathbb{Z}\langle\mathbf{a}, \mathbf{b}\rangle$ by letting $\Delta$ satisfy the following identities: $\Delta(1)=0, \Delta(\mathbf{a})=\Delta(\mathbf{b})=$ $1 \otimes 1$ and the Newtonian condition $\Delta(u \cdot v)=\Delta(u) \cdot v+u \cdot \Delta(v)$. For an ab-monomial $u=u_{1} u_{2} \cdots u_{n}$ we have that

$$
\Delta(u)=\sum_{i=1}^{n} u_{1} \cdots u_{i-1} \otimes u_{i+1} \cdots u_{n} .
$$

The following identity expresses the fundamental result that the ab-index is a coalgebra homomorphism [18].

Theorem 2.5 (Ehrenborg-Readdy) For a graded poset $P$ with ab-index $w=\Psi(P)$ and any $k$-multilinear map $M$ on $\mathbb{Z}\langle\mathbf{a}, \mathbf{b}\rangle$, the following coproduct identity holds:

$$
\sum_{c} M\left(\Psi\left(\left[x_{0}, x_{1}\right]\right), \Psi\left(\left[x_{1}, x_{2}\right]\right), \ldots, \Psi\left(\left[x_{k-1}, x_{k}\right]\right)\right)=\sum_{w} M\left(w_{(1)}, w_{(2)}, \ldots, w_{(k)}\right),
$$

where the first sum is over all chains $c=\left\{\hat{0}=x_{0}<x_{1}<\cdots<x_{k}=\hat{1}\right\}$ of length $k$ and the second sum is taken over the $k$-ary coproduct, that is, $\Delta^{k-1}$.

\subsection{The cd-index of the face poset of a central arrangement}

We recall the definition of the omega map [8].

Definition 2.6 The linear map $\omega$ from $\mathbb{Z}\langle\mathbf{a}, \mathbf{b}\rangle$ to $\mathbb{Z}\langle\mathbf{c}, \mathbf{d}\rangle$ is formed by replacing every occurrence of ab in a given $\mathbf{a b}-$ monomial by $2 \mathbf{d}$ and replacing the remaining letters by $\mathbf{c}$.

For a central hyperplane arrangement $\mathcal{H}$ the $\mathbf{c d}$-index of the face poset is computed as follows [8]:

Theorem 2.7 (Billera-Ehrenborg-Readdy) Let $\mathcal{H}$ be a central hyperplane arrangement with intersection lattice $\mathcal{L}$ and face lattice $T$. Then the cd-index of the face lattice $T$ is given by

$$
\Psi(T)=\omega(\mathbf{a} \cdot \Psi(\mathcal{L}))^{*} .
$$


We review the basic ideas behind the proof of this theorem. We will refer back to them when we prove similar results for toric and affine arrangements in Sections 3 and 4 .

Define two linear operators $\kappa$ and $\eta$ on $\mathbb{Z}\langle\mathbf{a}, \mathbf{b}\rangle$ by the following relations [8, Section 5]:

$$
\begin{aligned}
\kappa(\Psi(P)) & =(\mathbf{a}-\mathbf{b})^{\rho(P)-1}, \\
\eta(\Psi(P)) & =Z(P) \cdot(\mathbf{a}-\mathbf{b})^{\rho(P)-1} .
\end{aligned}
$$

For $k \geq 1$ the operator $\varphi_{k}$ is defined by the coalgebra expression

$$
\varphi_{k}(v)=\sum_{v} \kappa\left(v_{(1)}\right) \cdot \mathbf{b} \cdot \eta\left(v_{(2)}\right) \cdot \mathbf{b} \cdots \mathbf{b} \cdot \eta\left(v_{(k)}\right)
$$

where the coproduct splits $v$ into $k$ parts. Finally $\varphi$ is defined as the sum

$$
\varphi(v)=\sum_{k \geq 1} \varphi_{k}(v)
$$

Note that in this expression only a finite number of terms are non-zero. The connection with hyperplane arrangements is given by the following proposition.

Proposition 2.8 The ab-index of the lattice of faces of a central hyperplane arrangement is given by

$$
\Psi(T)=\varphi(\Psi(\mathcal{L} \cup\{\hat{0}\}))^{*} .
$$

Proposition 2.9 For an ab-monomial $w$ that begins with a, the two maps $\varphi$ and $\omega$ coincide, that is, $\varphi(w)=\omega(w)$.

Finally, Theorem 2.7 follows by Proposition 2.9 and from the fact that $\Psi(\mathcal{L} \cup\{\hat{0}\})=\mathbf{a} \cdot \Psi(\mathcal{L})$.

\subsection{Regular subdivisions of manifolds}

A regular subdivision of the sphere has an Eulerian face poset and hence a cd-index. For regular subdivisions of compact manifolds, a similar result holds. This was independently observed by Ed Swartz [41].

Theorem 2.10 Let $\Omega$ be a regular $C W$-complex whose geometric realization is a compact $n$-dimensional manifold $M$. Let $\chi(M)$ denote the Euler characteristic of $M$. Then the ab-index of the face poset $P$ of $\Omega$ has the following form.

(i) If $n$ is odd then $P$ is an Eulerian poset and hence $\Psi(P)$ can written in terms of $\mathbf{c}$ and $\mathbf{d}$.

(ii) If $n$ is even then $\Psi(P)$ has the form

$$
\Psi(P)=\left(1-\frac{\chi(M)}{2}\right) \cdot(\mathbf{a}-\mathbf{b})^{n+1}+\frac{\chi(M)}{2} \cdot \mathbf{c}^{n+1}+\Phi,
$$

where $\Phi$ is a homogeneous $\mathbf{c d}-$ polynomial of degree $n+1$ and where the term $\mathbf{c}^{n+1}$ does not occur.

For the $n$-dimensional torus Theorem 2.10 can be expressed as follows.

Corollary 2.11 Let $\Omega$ be a regular $C W$-complex whose geometric realization is the $n$-dimensional torus $T^{n}$. Then the ab-index of the face poset $P$ of $\Omega$ has the following form:

$$
\Psi(P)=(\mathbf{a}-\mathbf{b})^{n+1}+\Phi,
$$

where $\Phi$ is a homogeneous $\mathbf{c d}$-polynomial of degree $n+1$ and where the term $\mathbf{c}^{n+1}$ does not occur. 

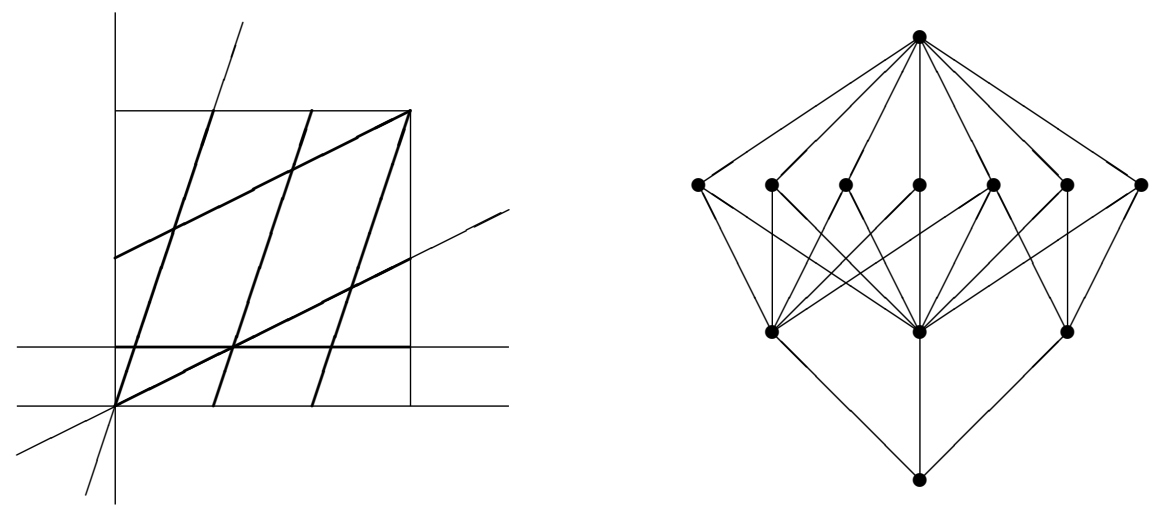

Fig. 1: A toric line arrangement and its intersection poset.

\section{Toric arrangements}

\subsection{Toric subspaces and arrangements}

The $n$-dimensional torus $T^{n}$ is defined as the quotient $\mathbb{R}^{n} / \mathbb{Z}^{n}$. Let $V$ be a $k$-dimensional affine subspace in $\mathbb{R}^{n}$ with rational coefficients. That is, $V$ has the form $V=\left\{\vec{v} \in \mathbb{R}^{n}: A \vec{v}=\vec{b}\right\}$, where the matrix $A$ has rational entries and the vector $\vec{b}$ is allowed to have real entries. Let $\bar{V}$ denote the image of $V$ under the quotient map $\mathbb{R}^{n} \rightarrow \mathbb{R}^{n} / \mathbb{Z}^{n}$. We call the image $\bar{V}$ a toric subspace of the torus $T^{n}$. When we remove the condition that the matrix $A$ is rational, the image is no longer homeomorphic to a torus. The intersection of two toric subspaces is in general not a toric subspace, but instead is the disjoint union of a finite number of toric subspaces. For two affine subspaces $V$ and $W$ with rational coefficients, we have that $\overline{V \cap W} \subseteq \bar{V} \cap \bar{W}$. This containment can be strict.

A toric hyperplane arrangement $\mathcal{H}=\left\{H_{1}, \ldots, H_{m}\right\}$ is a finite collection of toric hyperplanes. Define the intersection poset $\mathcal{P}$ of a toric arrangement to be the set of all connected components in all possible intersections of the toric hyperplanes, that is, all connected components of $\bigcap_{i \in S} H_{i}$ where $S \subseteq\{1, \ldots, m\}$, together with the empty set. We order the elements of the intersection poset $\mathcal{P}$ by reverse inclusion, that is, the torus $T^{n}$ is the minimal element of $\mathcal{P}$ corresponding to the empty intersection, and the empty set is the maximal element. A toric subspace $V$ is contained in the intersection poset $\mathcal{P}$ if there are toric hyperplanes $H_{i_{1}}, \ldots, H_{i_{k}}$ in the arrangement such that $V \subseteq H_{i_{1}} \cap \cdots \cap H_{i_{k}}$ and there is no toric subspace $W$ satisfying $V \subset W \subseteq H_{i_{1}} \cap \cdots \cap H_{i_{k}}$. In other words, $V$ has to be a maximal toric subspace in some intersection of toric hyperplanes from the arrangement.

The notion of using the intersection poset can be found in work of Zaslavsky, where he considers topological dissections [44]. In this setting there there is not an intersection lattice, but rather an intersection poset.

For a toric hyperplane arrangement $\mathcal{H}$ define the toric characteristic polynomial to be

$$
\chi(\mathcal{H} ; t)=\sum_{\substack{x \in \mathcal{P} \\ x \neq \emptyset}} \mu(\hat{0}, x) \cdot t^{\operatorname{dim}(x)}
$$

Example 3.1 Consider the line arrangement consisting of the three lines $y=3 \cdot x, x=2 \cdot y$ and $y=1 / 5$. It subdivides the torus into a regular $C W$-complex. The subdivision and the associated intersection poset are shown in Figure 1 . The characteristic polynomial is given by $\chi(\mathcal{H} ; t)=t^{2}-3 \cdot t+8$. Furthermore, the ab-index of the subdivision of the torus 
is given by $\Psi\left(T_{t}\right)=(\mathbf{a}-\mathbf{b})^{3}+7 \cdot \mathbf{d} \mathbf{c}+8 \cdot \mathbf{c d}$, as the following calculation shows.

\begin{tabular}{crrcccc}
$S$ & $f_{S}$ & $h_{S}$ & $u_{S}$ & $(\mathbf{a}-\mathbf{b})^{3}$ & $7 \cdot \mathbf{d c}$ & $8 \cdot \mathbf{c d}$ \\
\hline$\emptyset$ & 1 & 1 & aaa & 1 & 0 & 0 \\
$\{1\}$ & 7 & 6 & baa & -1 & 7 & 0 \\
$\{2\}$ & 15 & 14 & aba & -1 & 7 & 8 \\
$\{3\}$ & 8 & 7 & aab & -1 & 0 & 8 \\
$\{1,2\}$ & 30 & 9 & bba & 1 & 0 & 8 \\
$\{1,3\}$ & 30 & 16 & bab & 1 & 7 & 8 \\
$\{2,3\}$ & 30 & 8 & abb & 1 & 7 & 0 \\
$\{1,2,3\}$ & 60 & -1 & bbb & -1 & 0 & 0
\end{tabular}

Observe that the sum of the three last columns is equal to the flag $h$-vector.

We now give a natural interpretation of the toric characteristic polynomial. Let $G$ be the collection of finite intersections of toric subspaces of the $n$-dimensional torus $T^{n}$ together with the empty set. Observe that $T^{n}$ also belongs to $G$ and that $G$ is closed under finite intersections. Let $L$ be the distributive lattice consisting of all subsets of the torus $T^{n}$ that are obtained from the collection $G$ by finite intersections, finite unions and complements. The set $G$ is the generating set for the lattice $L$. A valuation $v$ is a function on the lattice $L$ such that $v(\emptyset)=0$ and $v(A)+v(B)=v(A \cap B)+v(A \cup B)$ for all sets $A, B \in L$. Similar to Theorem 2.1 in [19] we have:

Theorem 3.2 There is a valuation $v$ on the distributive lattice $L$ such that the valuation $v$ applied to a $k$-dimensional toric subspace $V$ is $t^{k}$, that is, $v(V)=t^{k}$.

By Möbius inversion we directly have the following theorem. The proof is standard. See the references [1, 10, 19, 28].

Theorem 3.3 The characteristic polynomial of a toric arrangement is given by

$$
\chi(\mathcal{H})=v\left(T^{n}-\bigcup_{i=1}^{m} H_{i}\right) .
$$

Observe that the Euler valuation of a $k$-dimensional torus is given by the Kronecker delta $\delta_{k, 0}$. This corresponds to setting $t=0$ in the valuation. Using that the Euler valuation of a $n$-dimensional region is $(-1)^{n}$, we have the next result. The proof is analogous to the proofs in [19, 20].

Theorem 3.4 Let $\mathcal{H}$ be a toric hyperplane arrangement on the $n$-dimensional torus $T^{n}$ that subdivides the torus into regions that are open $n$-dimensional balls. Then the number of regions of the arrangement is given by $(-1)^{n} \cdot \chi(\mathcal{H} ; 0)$.

Continuation of Example 3.1 Setting $t=0$ in the characteristic polynomial in Example 3.1 we obtain 8, which is indeed is the number of regions of this arrangement.

We call a toric hyperplane arrangement $\mathcal{H}=\left\{H_{1}, \ldots, H_{m}\right\}$ rational if each hyperplane $H_{i}$ is of the form $\vec{a}_{i} \cdot \vec{x}=b_{i}$ where the vector $\vec{a}_{i}$ has integer entries and $b_{i}$ is an integer. This is equivalent to assuming every constant $b_{i}$ is rational since every vector $\vec{a}_{i}$ was already assumed to be rational. In what follows we assume every coefficient is integral in a given rational arrangement. Define $M(\mathcal{H})$ to be the least common multiple of all the $n \times n$ minors of the $n \times m$ matrix $\left(\vec{a}_{1}, \ldots, \vec{a}_{m}\right)$. We can now give different interpretation of the toric chromatic polynomial by counting lattice points.

Theorem 3.5 For a rational hyperplane arrangement $\mathcal{H}$ there exists a constant $k$ such that for every $q>k$ and $q$ a multiple of $M(\mathcal{H})$, the toric characteristic polynomial evaluated at $q$ is given by the number of lattice points in $\left(\frac{1}{q} \mathbb{Z}\right)^{n} / \mathbb{Z}^{n}$ that do not lie on any of the toric hyperplanes $H_{i}$, that is,

$$
\chi(\mathcal{H} ; q)=\left|\left(\frac{1}{q} \mathbb{Z}\right)^{n} / \mathbb{Z}^{n}-\bigcup_{i=1}^{m} H_{i}\right| .
$$


The condition that $q$ is a multiple of $M(\mathcal{H})$ implies that every subspace $x$ in the intersection poset $\mathcal{P}$ intersects the toric lattice $\left(\frac{1}{q} \mathbb{Z}\right)^{n} / \mathbb{Z}^{n}$ in exactly $q^{\operatorname{dim}(x)}$ points. Theorem 3.5 now follows by Möbius inversion. This theorem is the toric analogue of Athanasiadis' finite field method. See especially [2, Theorem 2.1].

In the case when $M(\mathcal{H})=1$, the toric arrangement $\mathcal{H}$ is called unimodular. Novik, Postnikov and Sturmfels [36] state Theorem 3.4 in the special case of unimodular arrangements. Their first proof is based upon Zaslavksy's result on the number of regions in an affine arrangement. The second proof, due to Vic Reiner, is equivalent to our proof for arbitrary toric arrangements.

\subsection{The toric Bayer-Sturmfels result}

Define the toric Zaslavsky invariant of a graded poset $P$ by

$$
Z_{t}(P)=\sum_{x \text { coatom of } P}(-1)^{\rho(\hat{0}, x)} \cdot \mu(\hat{0}, x)=(-1)^{\rho(P)-1} \cdot \sum_{x \text { coatom of } P} \mu(\hat{0}, x) .
$$

We reformulate Theorem 3.4 as follows.

Theorem 3.6 For a toric hyperplane arrangement $\mathcal{H}$ on the torus $T^{n}$ that subdivides the torus into open n-dimensional balls, the number of regions is given by $Z_{t}(\mathcal{P})$, where $\mathcal{P}$ is the intersection poset of the arrangement $\mathcal{H}$.

For the remainder of this section we will assume that the subdivision of the torus induced by the toric arrangement is a regular $C W$-complex. Let $T_{t}$ denote the face poset of this subdivision. Define the map $z_{t}: T_{t}^{*} \longrightarrow \mathcal{P} \cup\{\hat{0}\}$ by sending each face to the smallest toric subspace in the arrangement that contains the face and sending the minimal element in $T_{t}^{*}$ to $\hat{0}$. Observe that the map $z_{t}$ is order- and rank-preserving, as well as being surjective.

The toric analogue of Theorem 2.3 is as follows.

Theorem 3.7 Let $P$ be the intersection poset of a toric hyperplane arrangement. Let $c=\left\{\hat{0}=x_{0}<x_{1}<\cdots<x_{k}=\hat{1}\right\}$ be a chain in $\mathcal{P} \cup\{\hat{0}\}$ with $k \geq 2$. Then the cardinality of the inverse image of the chain $c$ is given by the product

$$
\left|z_{t}^{-1}(c)\right|=\prod_{i=2}^{k-1} Z\left(\left[x_{i-1}, x_{i}\right]\right) \cdot Z_{t}\left(\left[x_{k-1}, x_{k}\right]\right)
$$

\subsection{The connection between posets and coalgebras}

For an ab-monomial $v$ define the linear map $\lambda_{t}$ by letting

$$
\lambda_{t}(v)= \begin{cases}(\mathbf{a}-\mathbf{b})^{m} & \text { if } v=\mathbf{b}^{m} \text { for some } m \geq 0 \\ (\mathbf{a}-\mathbf{b})^{m+1} & \text { if } v=\mathbf{b}^{m} \mathbf{a} \text { for some } m \geq 0 \\ 0 & \text { otherwise }\end{cases}
$$

The next lemma gives the relation between the toric Zaslavsky invariant $Z_{t}$ and the map $\lambda_{t}$.

Lemma 3.8 For a poset $P$, the following identity holds:

$$
\lambda_{t}(\Psi(P))=Z_{t}(P) \cdot(\mathbf{a}-\mathbf{b})^{\rho(P)-1} .
$$

Define a sequence of functions $\varphi_{t, k}: \mathbb{Z}\langle\mathbf{a}, \mathbf{b}\rangle \rightarrow \mathbb{Z}\langle\mathbf{a}, \mathbf{b}\rangle$ by $\varphi_{t, 1}=\kappa$, and for $k \geq 2$,

$$
\varphi_{t, k}(v)=\sum_{v} \kappa\left(v_{(1)}\right) \cdot \mathbf{b} \cdot \eta\left(v_{(2)}\right) \cdot \mathbf{b} \cdot \eta\left(v_{(3)}\right) \cdot \mathbf{b} \cdots \mathbf{b} \cdot \eta\left(v_{(k-1)}\right) \cdot \mathbf{b} \cdot \lambda_{t}\left(v_{(k)}\right) .
$$

Finally, let $\varphi_{t}(v)$ be the sum $\varphi_{t}(v)=\sum_{k \geq 1} \varphi_{t, k}(v)$. 
Theorem 3.9 The $\mathbf{a b}$-index of the face poset $T_{t}$ of a toric arrangement is given by

$$
\Psi\left(T_{t}\right)^{*}=\varphi_{t}(\Psi(\mathcal{P} \cup\{\hat{0}\})) .
$$

\subsection{Evaluating the function $\varphi_{t}$}

Define the linear operator $H^{\prime}$ on $\mathbb{Z}\langle\mathbf{a}, \mathbf{b}\rangle$ to be the one which removes the last letter in each monomial, that is, $H^{\prime}(w \cdot \mathbf{a})=$ $H^{\prime}(w \cdot \mathbf{b})=w$ and $H^{\prime}(1)=0$. We use the prime in the notation to distinguish it from the $H$ map defined in [8, Section 8] which instead removes the first letter in each ab-monomial.

Proposition 3.10 For an ab-monomial $v$ that begins with the letter a, the following holds:

$$
\varphi_{t}(v)=\kappa(v)+1 / 2 \cdot \omega\left(H^{\prime}(v) \cdot \mathbf{b}\right) .
$$

We now obtain the main result for computing the ab-index of the face poset of a toric arrangement.

Theorem 3.11 Let $\mathcal{H}$ be a toric hyperplane arrangement on the $n$-dimensional torus $T^{n}$ that subdivides the torus into a regular $C W$-complex. Then the ab-index of the face poset $T_{t}$ can be computed from the ab-index of the intersection poset $\mathcal{P}$ as follows:

$$
\Psi\left(T_{t}\right)=(\mathbf{a}-\mathbf{b})^{n+1}+\frac{1}{2} \cdot \omega\left(\mathbf{a} \cdot H^{\prime}(\Psi(\mathcal{P})) \cdot \mathbf{b}\right)^{*}
$$

Observe that in Proposition 3.10 and Theorem 3.11 no rational coefficients were introduced. Only the ab-monomial $\mathbf{a}^{n}$ is mapped to a cd-polynomial with an odd coefficient, hence $1 / 2 \cdot \omega(v \cdot \mathbf{b})$ has all integer coefficients.

Continuation of Example 3.1 The flag $f$-vector of the intersection poset $\mathcal{P}$ in Example 3.1 is given by $\left(f_{\emptyset}, f_{1}, f_{2}, f_{12}\right)=$ $(1,3,7,15)$, the flag $h$-vector by $\left(h_{\emptyset}, h_{1}, h_{2}, h_{12}\right)=(1,2,6,6)$, and so the $\mathbf{a b}$-index is $\Psi(P)=\mathbf{a}^{2}+2 \cdot \mathbf{b a}+6 \cdot \mathbf{a b}+6 \cdot \mathbf{b}^{2}$. Thus

$$
\begin{aligned}
\Psi\left(T_{t}\right) & =(\mathbf{a}-\mathbf{b})^{3}+1 / 2 \cdot \omega\left(\mathbf{a} \cdot H^{\prime}\left(\mathbf{a}^{2}+2 \cdot \mathbf{b a}+6 \cdot \mathbf{a b}+6 \cdot \mathbf{b}^{2}\right) \cdot \mathbf{b}\right)^{*} \\
& =(\mathbf{a}-\mathbf{b})^{3}+1 / 2 \cdot \omega(\mathbf{a} \cdot(7 \cdot \mathbf{a}+8 \cdot \mathbf{b}) \cdot \mathbf{b})^{*} \\
& =(\mathbf{a}-\mathbf{b})^{3}+7 \cdot \mathbf{d} \mathbf{c}+8 \cdot \mathbf{c d},
\end{aligned}
$$

which agrees with the calculation in Example 3.1

\section{The complex of unbounded regions}

The unbounded Zaslavsky invariant is defined by

$$
Z_{u b}(P)=Z(P)-2 \cdot Z_{b}(P) .
$$

As the name suggests, the number of unbounded regions in a non-central arrangement is given by this invariant. By taking the difference of the two statements in Theorem 2.2 part (ii), we have:

Lemma 4.1 For a non-central hyperplane arrangement $\mathcal{H}$ the number of unbounded regions is given by $Z_{u b}(\mathcal{L})$, where $\mathcal{L}$ is the intersection lattice of the arrangement $\mathcal{H}$. 

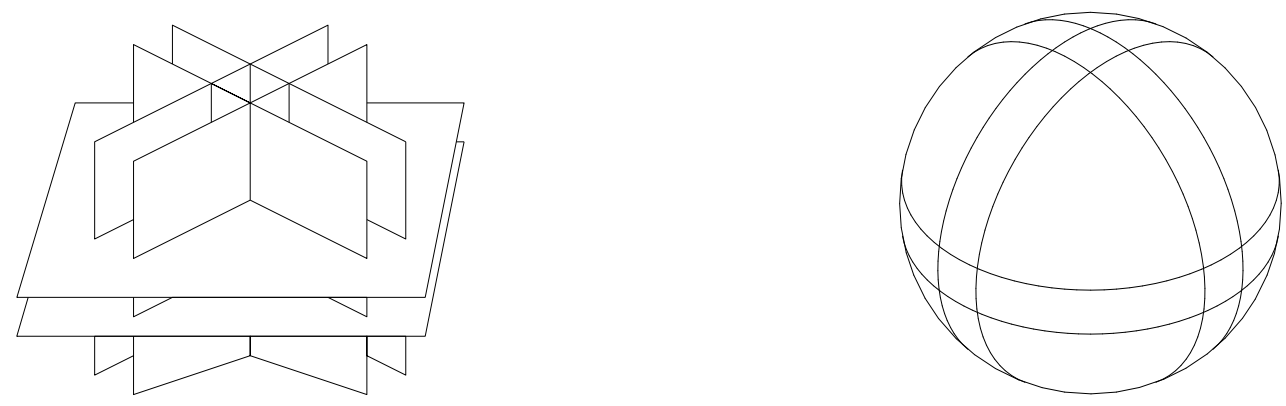

Fig. 2: The non-central arrangement $x, y, z=0,1$ and its associated spherical subdivision.

Let $\mathcal{H}$ be a non-central hyperplane arrangement in $\mathbb{R}^{n}$ with intersection lattice $\mathcal{L}$. Let $\mathcal{L}_{u b}$ denote the unbounded intersection lattice, that is, the subposet of the intersection lattice consisting of all affine subspaces with the points (dimension zero affine subspaces) omitted. Similarly, let $T_{u b}$ denote all of the faces in the hyperplane arrangement $\mathcal{H}$ which are unbounded. We observe that $T_{u b}$ is the face poset of an $(n-1)$-dimensional sphere. Pick $R$ large enough so that all of the bounded faces are strictly inside a ball of radius $R$. Intersect the arrangement $\mathcal{H}$ with a sphere of radius $R$. The resulting $C W$-complex has face poset $T_{u b}$. Our goal is to compute the cd-index of $T_{u b}$ in terms of the ab-index of $\mathcal{L}_{u b}$.

We now restrict the zero map $z: T^{*} \longrightarrow \mathcal{L} \cup\{\hat{0}\}$ to form the map $z_{u b}: T_{u b}^{*} \longrightarrow \mathcal{L} \cup\{\hat{0}\}$. Observe that $z_{u b}$ is order- and rank-preserving. Also note that $z_{u b}$ is not necessarily surjective. Analogous to the Bayer-Sturmfels result, Theorem 2.3 . we have the following theorem:

Theorem 4.2 Let $\mathcal{H}$ be a non-central hyperplane arrangement with intersection lattice $\mathcal{L}$. Let $c=\left\{\hat{0}=x_{0}<x_{1}<\right.$ $\left.\cdots<x_{k}=\hat{1}\right\}$ be a chain in $\mathcal{L} \cup\{\hat{0}\}$ with $k \geq 2$. Then the cardinality of its inverse image under $z_{u b}$ is given by

$$
\left|z_{u b}^{-1}(c)\right|=\prod_{i=2}^{k-1} Z\left(\left[x_{i-1}, x_{i}\right]\right) \cdot Z_{u b}\left(\left[x_{k-1}, x_{k}\right]\right) .
$$

We can now evaluate the cd-index of the poset of unbounded regions $T_{u b}$ in terms of the ab-index of the unbounded intersection lattice $\mathcal{L}_{u b}$.

Theorem 4.3 Let $\mathcal{H}$ be a non-central hyperplane arrangement with the unbounded intersection lattice $\mathcal{L}_{u b}$ and poset of unbounded regions $T_{u b}$. Then

$$
\Psi\left(T_{u b}\right)=\omega\left(\mathbf{a} \cdot \Psi\left(\mathcal{L}_{u b}\right)\right)^{*}
$$

Example 4.4 Consider the non-central hyperplane arrangement consisting of the six hyperplanes $x=0,1, y=0,1$ and $z=0,1$. Intersecting this with a sphere of large enough radius yields a spherical complex whose polytopal realization is the rhombicuboctahedron. The arrangement and its associated complex are displayed in Figure 2 . The dual of the face lattice of the spherical complex is not realized by a zonotope. However, the dual lattice can be viewed as the face lattice of a $2 \times 2 \times 2$ pile of cubes.

The intersection lattice $\mathcal{L}$ is the face lattice of the three-dimensional crosspolytope, in other words, the octahedron. Hence the lattice of unbounded intersection $\mathcal{L}_{u b}$ has the flag $f$-vector $\left(f_{\emptyset}, f_{1}, f_{2}, f_{12}\right)=(1,6,12,24)$ and the flag $h$ vector $\left(h_{\emptyset}, h_{1}, h_{2}, h_{12}\right)=(1,5,11,7)$. The $\mathbf{a b}$-index is given by $\Psi\left(\mathcal{L}_{u b}\right)=\mathbf{a}^{2}+5 \cdot \mathbf{b a}+11 \cdot \mathbf{a b}+7 \cdot \mathbf{b}^{2}$. Hence the cd-index of $T_{u b}$ is given by

$$
\begin{aligned}
\Psi\left(T_{u b}\right) & =\omega\left(\mathbf{a}^{3}+5 \cdot \mathbf{a b a}+11 \cdot \mathbf{a}^{2} \mathbf{b}+7 \cdot \mathbf{a b}^{2}\right)^{*} \\
& =\mathbf{c}^{3}+22 \cdot \mathbf{d} \mathbf{c}+24 \cdot \mathbf{c d} .
\end{aligned}
$$




\section{Concluding remarks}

For regular subdivisions of manifolds there is now a plethora of questions to ask.

(i) What is the right analogue of a regular subdivision in order that it be polytopal? Can flag $f$-vectors be classified for polytopal subdivisions?

(ii) Is there a Kalai convolution for manifolds that will generate more inequalities for flag $f$-vectors? [30]

(iii) Is there a lifting technique that will yield more inequalities for higher dimensional manifolds? [16]

(iv) Are there minimization inequalities for the cd-coefficients in the polynomial $\Psi$ ? As a first step, can one prove the non-negativity of $\Psi$ ? [7, 17]

(v) Is there an extension of the toric $g$-inequalities to manifolds? [4, 29, 31, 38]

(vi) Can the coefficients for $\Psi$ be minimized for regular toric arrangements as was done in the case of central hyperplane arrangements? [8]

The results in this extended abstract have been stated for hyperplane arrangements. In true generality one could work with the underlying oriented matroid, especially since there are nonrealizable ones such as the non-Pappus oriented matroid. All of these can be represented as pseudo-hyperplane arrangements. However, we have chosen to work with hyperplane arrangements in order not to lose the geometric intuition.

Other poset transformations that have been considered appear in [15, 22, 27]. Each uses a map related to the $\omega$ map. Are there toric or affine analogues of these posets transforms?

Another way to encode the flag $f$-vector data of a poset is to use the quasisymmetric function of a poset [13]. In this language the $\omega$ map is translated to Stembridge's $\vartheta$ map; see [9, 40]. Would the results of Theorems 3.11 and 4.3 be appealing in the quasisymmetric function viewpoint?

Richard Stanley has asked if the coefficients of the toric characteristic polynomial are alternating. If so, is there any combinatorial interpretation of the absolute values of the coefficients?

A far-reaching extension of Zaslavsky's results for complex hyperplane arrangements is by Goresky and MacPherson [24]. Their results determine the cohomology of the complement of a complex hyperplane arrangement. For a toric analogue of the Goresky-MacPherson results, see work of De Concini and Procesi [11]. For algebraic considerations of toric arrangements, see [12, 33, 34, 35].

In Section 3 we restricted ourselves to studying arrangements that cut the torus into regular $C W$-complexes. In a future paper [23], two of the authors are developing the notion of a cd-index for non-regular $C W$-complexes.

\section{Acknowledgements}

The authors thank the MIT Mathematics Department where this research was carried out. The first and third authors were partially supported by National Security Agency grant H98230-06-1-0072.

\section{References}

[1] C. A. Athanasiadis, Characteristic polynomials of subspace arrangements and finite fields, Adv. Math. 122 (1996), $193-233$.

[2] C. A. Athanasiadis, Extended Linial hyperplane arrangements for root systems and a conjecture of Postnikov and Stanley, $J$. Algebraic Combin. 10 (1999), 207-225.

[3] M. BAYER AND L. BillerA, Generalized Dehn-Sommerville relations for polytopes, spheres and Eulerian partially ordered sets, Invent. Math. 79 (1985), 143-157.

[4] M. Bayer And R. Ehrenborg, The toric $h$-vectors of partially ordered sets, Trans. Amer. Math. Soc. 352 (2000), $4515-4531$.

[5] M. Bayer And A. Klapper, A new index for polytopes, Discrete Comput. Geom. 6 (1991), 33-47. 
[6] M. Bayer And B. Sturmfels, Lawrence polytopes, Canad. J. Math. 42 (1990), 62-79.

[7] L. J. Billera And R. Ehrenborg, Monotonicity of the cd-index for polytopes, Math. Z. 233 (2000), 421-441.

[8] L. J. Billera, R. Ehrenborg, And M. Readdy, The c-2d-index of oriented matroids, J. Combin. Theory Ser. A 80 (1997), 79-105.

[9] L. J. Billera, S. K. Hsiao and S. van Willigenburg, Peak quasisymmetric functions and Eulerian enumeration, Adv. Math. 176 (2003), 248-276.

[10] B. CHEN, On characteristic polynomials of subspace arrangements, J. Combin. Theory Ser. A 90 (2000), 347-352.

[11] C. De Concini And C. Procesi, On the geometry of toric arrangements, Transform. Groups 10 (2005), $387-422$.

[12] J. M. Douglass, Toral arrangements and hyperplane arrangements, Rocky Mountain J. Math. 28 (1998), 939-956.

[13] R. Ehrenborg, On posets and Hopf algebras, Adv. Math. 119 (1996), 1-25.

[14] R. Ehrenborg, $k$-Eulerian posets, Order. 18 (2001), 227-236.

[15] R. Ehrenborg, The $r$-signed Birkhoff transform, submitted to Trans. Amer. Math. Soc.

[16] R. Ehrenborg, Lifting inequalities for polytopes, Adv. Math. 193 (2005), 205-222.

[17] R. Ehrenborg And K. KARU, Decomposition theorem for the cd-index of Gorenstein* posets, J. Algebraic Combin. 26 (2007), 225-251.

[18] R. Ehrenborg And M. Readdy, Coproducts and the cd-index, J. Algebraic Combin. 8 (1998), $273-299$.

[19] R. EhrEnBorg AND M. REAdDY, On valuations, the characteristic polynomial and complex subspace arrangements, Adv. Math. 134 (1998), 32-42.

[20] R. Ehrenborg AND M. Readdy, The Dowling transform of subspace arrangements, J. Combin. Theory Ser. A 91 (2000), 322-333.

[21] R. Ehrenborg And M. Readdy, Homology of Newtonian coalgebras, European J. Combin. 23 (2002), 919-927.

[22] R. Ehrenborg And M. Readdy, The Tchebyshev transforms of the first and second kind, to appear in Ann. Comb.

[23] R. Ehrenborg AND M. SlOne, The cd-index of non-regular $C W$-complexes, in preparation.

[24] M. Goresky AND R. MACPherson, "Stratified Morse theory" Ergebnisse der Mathematik und ihrer Grenzgebiete (3) [Results in Mathematics and Related Areas (3)], 14. Springer-Verlag, Berlin, 1988.

[25] H. Groemer, On the extension of additive functionals on classes of convex sets, Pacific J. Math. 75 (1978), $397-410$.

[26] B. GRÜNBAuM, “Convex polytopes," 2nd edition, Springer-Verlag, New York, 2003.

[27] S. K. Hsiao, A signed analog of the Birkhoff transform, J. Combin. Theory Ser. A 113 (2006), 251-272.

[28] T. JózeFiAK AND B. SAgAN, Basic derivations for subarrangements of Coxeter arrangements, J. Algebraic Combin. 2 (1993), 291-320.

[29] G. KalaI, Rigidity and the lower bound theorem. I, Invent. Math. 88 (1987), 125-151.

[30] G. Kalai, A new basis of polytopes, J. Combin. Theory Ser. A 49 (1988), 191-209.

[31] K. KARU, Hard Lefschetz theorem for nonrational polytopes, Invent. Math. 157 (2004), 419-447.

[32] D. Klain And G.-C. Rota, "Introduction to geometric probability," Lezioni Lincee, Cambridge University Press, Cambridge, 1997.

[33] C. Macmeikan, The Poincaré polynomial of an mp arrangement, Proc. Amer. Math. Soc. 132 (2004), 1575-1580.

[34] C. Macmeikan, Modules of derivations for toral arrangements, Indag. Math. (N.S.) 15 (2004), 257-267.

[35] C. Macmeikan, Toral arrangements. The COE Seminar on Mathematical Sciences 2004, 37-54, in Sem. Math. Sci., 31, Keio Univ., Yokohama, 2004.

[36] I. Novik, A. Postnikov And B. Sturmfels, Syzygies of oriented matroids, Duke Math. J. 111 (2002), $287-317$.

[37] R. P. Stanley, "Enumerative Combinatorics, Vol. I,” Wadsworth and Brooks/Cole, Pacific Grove, 1986.

[38] R. P. STANLEY, Generalized $h$-vectors, intersection cohomology of toric varieties, and related results. Commutative algebra and combinatorics (Kyoto, 1985), 187-213, Adv. Stud. Pure Math., 11, North-Holland, Amsterdam, 1987.

[39] R. P. Stanley, Flag $f$-vectors and the $c d$-index, Math. Z. 216 (1994), 483-499.

[40] J. Stembridge, Enriched P-partitions, Trans. Amer. Math. Soc. 349 (1997), 763-788.

[41] E. SWARTZ, Face enumeration — from spheres to manifolds. arXiv:0709.3998v1 [math.CO], preprint 2007.

[42] M. SweEdLER, "Hopf Algebras," Benjamin, New York, 1969.

[43] T. Zaslavsky, Facing up to arrangements: face count formulas for partitions of space by hyperplanes, Mem. Amer. Math. Soc. 154 (1975).

[44] T. Zaslavsky, A combinatorial analysis of topological dissections, Adv. Math. 25 (1977), 267-285. 